\title{
$\bullet$ IJCRR \\ Synthesis and in vitro Antibacterial, Antitubercular and Cytotoxicity Evaluation of Lomefloxacin Derivatives
}

Section: Healthcare ISI Impact Factor (2019-20): 1.628 IC Value (2019): 90.81 $\operatorname{SJIF}(2020)=7.893$

\author{
Gurunani Gulshan' ${ }^{*}$, Agrawal Kapil2, Walde Sheelpriya3 ${ }^{3}$, Ittadwar Abhay ${ }^{4}$
}

\begin{abstract}
'Research Scholar, Department of Pharmaceutical Chemistry, Gurunanak College of Pharmacy, Nagpur, 440026, India; ${ }^{2}$ Associate Professor, Department of Pharmaceutical Chemistry, R. C. Patel Institute of Pharmaceutical Education and Research, Shirpur, Dist. Dhule, 425405, India; 3Professor, Department of Pharmaceutical Chemistry, Gurunanak College of Pharmacy, Nagpur, 440026, India; 4Principal, Gurunanak College of Pharmacy, Nagpur, 440026, India.
\end{abstract}

\section{ABSTRACT}

Introduction: The fluoroquinolones antibacterial agents are one of the fastest growing groups of drugs in recent years. The various side chains on it can be altered and the resulting analogues are evaluated for their anti-microbial and antitubercular properties. Most of these agents are substituted at the 7 positions by nitrogen heterocycles. Lomefloxacin at C-7, which represents a site amenable to significant modification.

Objective: Based on evidence of research results and in search of new bioactive molecules in the fluoroquinolones, a of $\mathrm{N}$ substituted piperazinyl quinolones have been designed, synthesized, characterized and evaluated for their antibacterial activity and antitubercular activity.

Method: A series of 2-((5-chloro-1, 3, 4-thiadiazol-2yl) thio)-1-(4-subs.) ethanone (4a-4j)were prepared by diazotization of amines $(3 a-3 j)$ in concentrated $\mathrm{HCl}$ in the presence of $\mathrm{Cu}$-powder. The reaction of $(4 \mathrm{a}-4 \mathrm{j})$ with piperazinyl quinolone (lomefloxacin) in DMF yield (5a-5j). The synthesized compounds were evaluated against some Gram-positive and Gram-negative bacterias and antitubercular activity against Mtb WT H37Rv.

Result: The structure of the synthesized compound was confirmed by their IR, 1HNMR, data. The antibacterial data revealed that all substituted derivatives ( $5 \mathrm{a}$ to $5 \mathrm{j}$ ), are found to be least active against Gram-positive and Gram-negative organisms. Among all of the tested compounds, $5 \mathrm{~b}$ (Lomefloxacin derivative) exhibited excellent antitubercular activity against Mtb WT H37Rv (MIC0.8 $\mu \mathrm{g} / \mathrm{ml}$ ) which is comparable to that of standard. (MIC $0.8 \mu \mathrm{g} / \mathrm{ml}$ )

Conclusion: Although the nature of the C-7 substituent is known to enhance quinolone activity in bacteria but results of the present study reveal that the synthesized derivative shows significant antitubercular property but poor antibacterial activity.

Key Words: Antibacterial activity, Antitubercular activity, Fluoroquinolone, Lomefloxacin, N-piperazinyl quinolone, Synthesis

\section{INTRODUCTION}

Fluoroquinolones, a major class of antibiotics, are under clinical development. The antibacterial activity of Fluoroquinolones is due to the inhibition of bacterial enzymes; DNA-gyrase and topoisomerase IV. They have potent activity, rapid bactericidal effects, and a low prevalence of resistance development. ${ }^{1}$ The fluoroquinolones exert certain adverse effects, have restricted activity against Grampositivepathogens and methicillin-resistant Staphylococcus aureus (MRSA). ${ }^{2}$ Therefore, there is a need of synthesizing novel quinolones with better activity profile, pharmacokinetics, and acceptability, to overcome the limitations of existing drugs. ${ }^{3}$ Most of the quinolone antibacterial research has been focused on substitution at the C-7 as it is the most adaptable site for chemical change.C-7 position is an area that determines potency and target preference and also controls the pharmacokinetic properties of the drugs, with basic nitrogen. ${ }^{4-6}$ The most commonly found substitution at the C-7 position is a five- or six-membered ring. For example, aminopyrrolidine substituent at $\mathrm{C}-7$ in trovafloxacinandgemifloxacinandPiperazine substitution at the C-7 position in norfloxacin, ciprofloxacin, pefloxacin, pefloxacin, ofloxacin, amifloxacin, fleroxacin, lomefloxacin, sparfloxacin, difloxacin, enoxacin, enrofloxacin, levofloxacin, marbofloxacin, and orbifloxacin which has triggered a

\section{Corresponding Author:}

Gurunani Gulshan, Research Scholar, Department of Pharmaceutical Chemistry, Gurunanak College of Pharmacy, Nagpur, 440026, India; E-mail: gurugu127@gmail.com

ISSN: 2231-2196 (Print) ISSN: 0975-5241 (Online)

Received: $10.03 .2021 \quad$ Revised: 03.05 .202

Accepted: 12.06 .2021

Published: 09.11.2021 
wide range of clinically useful fluoroquinolone antibacterial agents. ${ }^{7-17}$ (Figure 1) The site near the C-7 substituent is regarded as the domain for drug-enzyme interaction and the cell permeability. ${ }^{18-21}$ The piperazine moiety of 7-piperazinyl quinolones possesses enough structural flexibility to allow product optimization. In the present study, we have aimed to achieve a better antimicrobial profile at a lower concentration, by preparing [(7-(4--(5-substituted-benzoylthio)-1,3,4thiadiazol-2-yl)-3-methylpiperazine-1-yl)-1-ethyl-6,8-difluoro-4-oxo-1,4-dihydroquinoline-3-carboxylic acid](5a to $5 \mathrm{j})$ derivatives and have been evaluated for its in vitro antibacterial and anti-tubercular effect. (Figure 2).

\section{MATERIALS AND METHODS}

\section{Materials}

All the chemicals, reagents, and solvents used in this research were bought from E Merck Ltd, Loba chemicals Ltd, SigmaAldrich Ltd., Spectrochem Ltd., Hi-media, and Rankem Chemicals Ltd. Mumbai, India. Solvents used were dried and purified as and when required. The melting points reported were uncorrected and were determined in open capillaries using Thiele's melting point apparatus and measured in $\left({ }^{\circ} \mathrm{C}\right)$. The yields of synthesized compounds were mentioned in tables along with respective physical constants. The FTIR spectra were obtained Shimadzu FTIR spectrophotometer and values were measured in $\mathrm{cm}^{-1}$ (potassium bromide disks). ${ }^{1} \mathrm{HNMR}$ and ${ }^{13} \mathrm{C}$ NMR were recorded at $400 \mathrm{MHz}$ and $100 \mathrm{MHz}$ respectively on a Bruker AM spectrometer, IISc Bangalore, and chemical shifts are expressed as $\delta(\mathrm{ppm})$ with tetramethylsilane as an internal standard. The FAB / EIMS mass spectra were recorded on Autospec Mass spectrometer, IICT, Hyderabad.

\section{Methods}

General Procedure for Synthesis of 2(a-j) (Figure 3)

Synthesis of substituted/unsubstituted phenacyl bromide $2(a-j)$

$0.1 \mathrm{~mol}$ of substituted/un-substituted acetophenones $1(\mathrm{a}-\mathrm{j})$ were taken in the two-necked round bottom flask, suitable anhydrous solvents (ether, acetone, methanol, chloroform) was added with anhydrous $\mathrm{AlCl} 3$. The reaction condition was kept up either in cold or at room temperature and bromine $(0.09 \mathrm{~mol})$ was added with stirring. Mixtures $2(\mathrm{a}-\mathrm{j})$ were acquired as colourless to brown to shining crystals. The product was washed twice with appropriate solvents and recrystallized from methanol to get lachrymatory crystals. ${ }^{22-23}$ Liquefying point ranges of 2a-j; $\mathrm{R}=\mathrm{H}, \mathrm{Cl}, \mathrm{Br}, \mathrm{F}, \mathrm{NO} 2, \mathrm{CH} 3$, OCH3, NH2, OH, C6H5; 48-50 ${ }^{\circ}, 90-92^{\circ}, 110-112^{\circ}, 46-48$, 96-98 $, 52-54^{\circ}, 72-74^{\circ}, 80-84^{\circ}, 102-104,98-102$ respectively (50-74\%).

General Procedure for Synthesis of $(a-j)$. (Figure 3)
Synthesis of 2-((amino-1,3,4-thiadiazol-2yl)thio)-1-(4-subst.) ethanone $3(a-j)$.

The 2-amino-5- mercapto-1,3,4-thiadiazole $(0.1 \mathrm{~mol})$ was suspended in $15 \mathrm{ml}$ of water and $80 \%$ potassium hydroxide $(0.1 \mathrm{~mol})$ was added. This solution was de-colorized with activated charcoal, followed by the addition of $32 \mathrm{~mL}$ of ethanol and stirred rapidly with $2(\mathrm{a}-\mathrm{j})(0.1 \mathrm{~mol})$. The reaction mixture was cooled for 40 minutes and it added $200 \mathrm{~mL}$ of cold water. It is then filtered to obtain the solid product and washed with ether and water. The $3(\mathrm{a}-\mathrm{j})$ were obtained (Scheme 1), with 54-68\% yield and melting point $\left(80-108^{\circ} \mathrm{C}\right) .^{24-25}$

General Procedure for Synthesis of $4(\mathrm{a}-\mathrm{j})$. (Figure 3)

Procedure for Synthesis of 2-((5-chloro-1, 3, 4-thiadiazol2yl) thio)-1-(4-subs.)ethanone4 (a-j)

Triturated 2-((amino-1,3,4-thiadiazol-2yl) thio)-1-(4-subs.) ethanone $3(\mathrm{a}-\mathrm{j})(30 \mathrm{mmol})$ with sodium nitrite $(60 \mathrm{mmol})$. The triturate was introduced in the ice-cooled $\left(0-5^{\circ} \mathrm{C}\right)$ mixture of $15 \mathrm{ml}$ water and $30 \mathrm{ml}$ concentrated $\mathrm{HCl}$ with stirring in the presence of copper powder. The product was refluxed for 1 hour at $75^{\circ} \mathrm{C}$ and cool. Then the mixture was extracted thrice with dry chloroform $(75 \mathrm{ml})$. The combined extracts of chloroform were washed with a sodium bicarbonate solution. Then the solution was dried over sodium sulphate followed by evaporation under reduced pressure. Finally, recrystallization of the product was done using ethanol to yield 2-((5-chloro-1, 3, 4-thiadiazol-2yl)thio)-1-(4-subst) ethanone $4(a-j)$ (Scheme 1). The compound was purified by column chromatography with methanol: chloroform (1:9) as mobile phase ${ }^{26}$, m.p. $85-110^{\circ} \mathrm{C}(48-60 \%)$.

General Procedure for Synthesis of 5(a-j). (Figure 3)

Synthesis of 1-subst.-6-fluoro-8-subst.-7-(3-subst.-4-(5subst.((2-oxo-2-(p-subst.)ethyl)thio)-1,3,4-thiadiazol-2-yl) piperazin-1-yl)-4-oxo-1,4-dihydroquinoline-3-carboxylic acid $5(\mathrm{a}-\mathrm{j})$.

A combination of equimolar quantities of compound 2-((5-chloro-1, 3, 4-thiadiazol-2yl)thio)-1-(4-subs.) ethanone $4(\mathrm{a}-\mathrm{j})$ and piperazinyl fluoroquinolone (sparfloxacin), along with sodium-bicarbonate in $10 \mathrm{ml}$ dimethyl-formamide was refluxed on an oil bath at $140-160^{\circ} \mathrm{C}$ for hrs. After cooling the reaction mixture, $10 \mathrm{ml}$ of cold water was added to it. The precipitated product was filtered and washed with water. The product was then subjected to recrystallization using a blend of dimethylformamide and water to yield (5a-j) compounds. ${ }^{27-28}$ (Scheme 1). The Physicochemical results are shown in (Table 1)

\section{Antibacterial Activity}

Preliminary in vitro antibacterial activity was employed by the broth micro-dilution technique. Antibacterial Activity 
was examined against two Gram-negative microorganisms, Pseudomonas aeruginosa and Escherichia coli, and two Gram-positive microorganisms, Staphylococcus aureus and Bacillus subtilis. The test compounds and reference drugs (Sparfloxacin and Rifampicin) were prepared in MuellerHinton agar medium by two-fold serial dilutions. The required concentrations of $0.5,1.0,2.5,5.0,7.5,10.0,12.5$, $15.0,17.5$, and $20.0 \mu \mathrm{g} / \mathrm{ml}$ was obtain by Progressive double dilutions with agar. The Petri plates were inoculated with $1-5 \times 10^{4}$ colonies forming units $(\mathrm{CFU} / \mathrm{ml})$ and incubated at $37^{\circ} \mathrm{C}$ for 18 hours. ${ }^{29}$ The results are presented in (Table 2).

\section{Anti-tubercular Activity}

In vitro screening for anti-mycobacterial was performed by utilizing M. tuberculosis virulent H37Rv strain. The broth dilution assay for each drug for determination of MIC was determined by using the frozen culture of Middlebrook $7 \mathrm{H} 9$ broth supplemented with $10 \%$ ADC (albumin dextrose catalase) and $0.2 \%$ glycerol. It is used as inoculum with dilution in broth to $2 \times 10^{5} \mathrm{CFU} / \mathrm{ml}$. In the assay, for the accommodation of compounds U-tubes were used in $0.1,0.5,1.5,2.5$, $05,7.5,10,12.5,15,17.5$ and $20 \mu \mathrm{g} / \mathrm{ml}$ dilutions. ${ }^{30-31}$ The results are presented in (Table 2).

\section{In-vitro cytotoxic study}

\section{Estimation of cell viability}

Conversion of MTT [(3-(4,5-dimethyl thiazol-2-yl)-2,5-diphenyl tetrasodium bromide)] to dark blue formazan crystals due to the presence of living cells, was employed for estimation of cell viability. Colourimetric analysis was used for the estimation of MTT cleaved to the viable cells. The solution of compounds under investigation in DMSO was diluted to achieve test concentrations. The DMSO content was maintained below $0.1 \%$ in all the aliquots under investigation. The cultured Hep-G2 normal liver-cell lines were added in plates with 96 wells and then preserved with variable dilutions of investigational compounds in $\mathrm{DMSO}$, at $37^{\circ} \mathrm{C}$ in a carbon dioxide incubator for four days. Further, the MTT reagent was instilled into the wells and incubated for four hours, and then the dark blue formazan developed was allowed to dissolve in DMSO and the colourimetric absorbance was read at 550 $\mathrm{nm}$. The $\mathrm{IC}_{50}$ value was estimated by graph plotted between percentage cells inhibited versus concentrations. ${ }^{32}$ The findings are provided in (Table 2).

\section{RESULTS}

\section{Spectral Data of synthesized compound}

1-ethyl-6,8-difluoro-7-(3-methyl-4-(5-((2-oxo-2-phenylethyl)thio)-1,3,4-thiadiazol-2-yl)piperazin-1-yl)-4-oxo-1,4dihydroquinoline-3-carboxylic acid (5a)
IR (KBr) cm ${ }^{-1}$ : 3422(carboxylic, O-H str.), 2943(Ar. C-H str.), 2856(Ali. $\mathrm{CH}_{2}, \mathrm{C}-\mathrm{H}$ str.), 1716 (carboxylic, $\mathrm{C}=\mathrm{O}$ str.), 1642(ketonic, $\mathrm{C}=\mathrm{O}$ str.), 1588(Imine, $\mathrm{C}=\mathrm{N}$ str.), 1320(ethylic, C-H str.); ${ }^{1} \mathrm{H}-\mathrm{NMR}$ (DMSO-d6) $\delta p p m: 12.52$ (s, carboxylic, $1 \mathrm{H}, \mathrm{OH}), 9.02(\mathrm{~s}, 1 \mathrm{H}, \mathrm{H} 2$-quinoline), 7.56-7.94(m, 5H, Ar.), 7.58(s, 1H, H5-quinoline), 4.92(s, 2H, $\left.\mathrm{CH}_{2}\right), 4.64(\mathrm{q}$, $\left.2 \mathrm{H}, \mathrm{NCH}_{2} \mathrm{CH}_{3}\right), 2.92-3.50(\mathrm{~m}, 7 \mathrm{H}$, piperazinyl), $1.38(\mathrm{t}, 3 \mathrm{H}$, $\left.\mathrm{NCH}_{2} \mathrm{CH}_{3}\right), 1.29\left(\mathrm{~s}, 3 \mathrm{H}\right.$, piperazinyl $\left.\mathrm{CH}_{3}\right)$.

\section{7-(4-(5-((2-(4-chlorophenyl)-2-oxoethyl)thio)-}

1,3,4-thiadiazol-2-yl)-3-methylpiperazin-1-yl)1-ethyl-6,8-difluoro-4-oxo-1,4-dihydroquinoline3-carboxylic acid (5b)

IR (KBr) $\mathrm{cm}^{-1}$ : 3445(carboxylic, O-H str.), 3005(Ar. C-H str.), 2852(Ali. $\mathrm{CH}_{2}, \mathrm{C}-\mathrm{H}$ str.), 1725(carboxylic, $\mathrm{C}=\mathrm{O}$ str.), 1656(ketonic, $\mathrm{C}=\mathrm{O}$ str.), 1583(Imine, $\mathrm{C}=\mathrm{N}$ str.), 1307(ethylic, C-H str.); ${ }^{1} \mathrm{H}-\mathrm{NMR}$ (DMSO-d6) $\delta p p m: 12.55$ (s, carboxylic, $1 \mathrm{H}, \mathrm{OH}), 8.91(\mathrm{~s}, 1 \mathrm{H}, \mathrm{H} 2$-quinoline), 7.60-7.92(m, $4 \mathrm{H}$, Ar.), 7.42(s, 1H, H5-quinoline), 4.84(s, 2H, $\left.\mathrm{CH}_{2}\right), 4.57$ (q, $\left.2 \mathrm{H}, \mathrm{NCH}_{2} \mathrm{CH}_{3}\right), 2.94-3.47(\mathrm{~m}, 7 \mathrm{H}$, piperazinyl), $1.46(\mathrm{t}, 3 \mathrm{H}$, $\mathrm{NCH}_{2} \mathrm{CH}_{3}$ ), $1.33\left(\mathrm{~s}, 3 \mathrm{H}\right.$, piperazinyl $\left.\mathrm{CH}_{3}\right) ;{ }^{13} \mathrm{C}-\mathrm{NMR}$ (DMSOd6) Sppm: 198, 180, 161, 158, 148, 144, 130, 118, 110, 68, 40, 18; MS: $\mathrm{m} / \mathrm{z}=619\left[\mathrm{M}^{+}\right] ; \mathrm{CHN}$ calcd; $\mathrm{C}_{27} \mathrm{H}_{24} \mathrm{ClF}_{2} \mathrm{~N}_{5} \mathrm{O}_{4} \mathrm{~S}$; $\mathrm{C}$, 52.30; H, 3.90; N, 11.29; Found C, 52.34; H, 3.90; N, 11.30.

7-(4-(5-((2-(4-bromophenyl)-2-oxoethyl)thio)1,3,4-thiadiazol-2-yl)-3-methylpiperazin-1-yl)1-ethyl-6,8-difluoro-4-oxo-1,4-dihydroquinoline3-carboxylic acid (5c)

IR (KBr) $\mathrm{cm}^{-1}$ : 3444(carboxylic, O-H str.), 3009(Ar. C-H str.), 2850(Ali. $\mathrm{CH}_{2}, \mathrm{C}-\mathrm{H}$ str.), 1728(carboxylic, $\mathrm{C}=\mathrm{O}$ str.), 1625(ketonic, $\mathrm{C}=\mathrm{O}$ str.), 1554(Imine, $\mathrm{C}=\mathrm{N}$ str.), 1310(ethylic, C-H str.); ${ }^{1} \mathrm{H}-\mathrm{NMR}$ (DMSO-d6) $\delta p p m: 12.58(\mathrm{~s}$, carboxylic, $1 \mathrm{H}, \mathrm{OH}), 8.93(\mathrm{~s}, 1 \mathrm{H}, \mathrm{H} 2$-quinoline), 7.61-7.94(m, $4 \mathrm{H}$, Ar.), 7.44(s, 1H, H5-quinoline), 4.80(s, 2H, $\left.\mathrm{CH}_{2}\right), 4.59(\mathrm{q}$, $\left.2 \mathrm{H}, \mathrm{NCH}_{2} \mathrm{CH}_{3}\right), 2.93-3.48(\mathrm{~m}, 7 \mathrm{H}$, piperazinyl), $1.48(\mathrm{t}$, $\left.3 \mathrm{H}, \mathrm{NCH}_{2} \mathrm{CH}_{3}\right), 1.31\left(\mathrm{~s}, 3 \mathrm{H}\right.$, piperazinyl, $\left.\mathrm{CH}_{3}\right) ;{ }^{13} \mathrm{C}-\mathrm{NMR}$ (DMSO-d6) Sppm: 199, 181, 165, 160, 149, 128, 120, 108, $70,42,17$.

\subsection{7-(4-(5-((2-(4-fluorophenyl)-2-oxoethyl)thio)- 1,3,4-thiadiazol-2-yl)-3-methylpiperazin-1-yl)- 1-ethyl-6,8-difluoro-4-oxo-1,4-dihydroquinoline- 3-carboxylic acid (5d)}

IR (KBr) cm$^{-1}$ : 3454(carboxylic, O-H str.), 2926(Ar. C-H str.), 2853(Ali. $\mathrm{CH}_{2}, \mathrm{C}-\mathrm{H}$ str.), 1726(carboxylic, $\mathrm{C}=\mathrm{O}$ str.), 1658(ketonic, $\mathrm{C}=\mathrm{O}$ str.), 1584(Imine, $\mathrm{C}=\mathrm{N}$ str.), 1327 (ethylic, C-H str.); ${ }^{1} \mathrm{H}-\mathrm{NMR}$ (DMSO-d6) $\delta p p m: 12.68(\mathrm{~s}$, carboxylic, $1 \mathrm{H}, \mathrm{OH}), 8.94(\mathrm{~s}, 1 \mathrm{H}, \mathrm{H} 2$-quinoline), 7.988.02(m, 4H, Ar.), 7.78(s, 1H, H5-quinoline), 4.59(s, 2H, $\left.\mathrm{CH}_{2}\right), 4.42\left(\mathrm{q}, 2 \mathrm{H}, \mathrm{NCH}_{2} \mathrm{CH}_{3}\right), 2.50-3.50(\mathrm{~m}, 7 \mathrm{H}$, piperazinyl), 1.48(t, $\left.3 \mathrm{H}, \mathrm{NCH}_{2} \mathrm{CH}_{3}\right), 1.25\left(\mathrm{~s}, 3 \mathrm{H}\right.$, piperazinyl $\left.\mathrm{CH}_{3}\right)$; ${ }^{13} \mathrm{C}-\mathrm{NMR}(\mathrm{DMSO}-d 6)$ Sppm: 197, 173, 148, 133, 117, 
107, 98, 74, 48, 38, 37; MS: m/z = $602\left[\mathrm{M}^{+}\right]$; CHN calcd; $\mathrm{C}_{27} \mathrm{H}_{24} \mathrm{~F}_{3} \mathrm{~N}_{5} \mathrm{O}_{4} \mathrm{~S}_{2} ; \mathrm{C}, 53.72 ; \mathrm{H}, 4.01 ; \mathrm{N}, 11.60$; Found $\mathrm{C}$, $54.12 ; \mathrm{H}, 3.98 ; \mathrm{N}, 11.94$.

\section{7-(4-(5-((2-(4-nitrophenyl)-2-oxoethyl)thio)-} 1,3,4-thiadiazol-2-yl)-3-methylpiperazin-1-yl)1-ethyl-6,8-difluoro-4-oxo-1,4-dihydroquinoline3-carboxylic acid (5e)

IR (KBr) $\mathrm{cm}^{-1}$ : 3441(s, carboxylic, O-H str.), 3006(Ar. $\mathrm{C}-\mathrm{H}$ str.), 1374(Ali. $\mathrm{CH}_{2}, \mathrm{C}-\mathrm{H}$ str.), 1728(carboxylic, $\mathrm{C}=\mathrm{O}$ str.), 1624(ketonic, $\mathrm{C}=\mathrm{O}$ str.), 1547(Imine, $\mathrm{C}=\mathrm{N}$ str.), 1311(ethylic, C-H str.); ${ }^{1} \mathrm{H}-\mathrm{NMR}$ (DMSO-d6) $\delta p p m$ : 12.56(s, 1H, carboxylic, OH), 8.92(s, 1H, H2-quinoline), 7.74-7.92(m, 4H, Ar.), 7.47(s, 1H, H5-quinoline), 4.83(s, $\left.2 \mathrm{H}, \mathrm{CH}_{2}\right), 4.65\left(\mathrm{q}, 2 \mathrm{H}, \mathrm{NCH}_{2} \mathrm{CH}_{3}\right), 2.92-3.47(\mathrm{~m}, 7 \mathrm{H}$, piperazinyl), $1.47\left(\mathrm{t}, 3 \mathrm{H}, \mathrm{NCH}_{2} \mathrm{CH}_{3}\right), 1.38(\mathrm{~s}, 3 \mathrm{H}$, piperazinyl $\mathrm{CH}_{3}$ ); ${ }^{13} \mathrm{C}-\mathrm{NMR}$ (DMSO-d6) Sppm: 192, 183, 165, 155, $147,138,129,113,74,47,11 ; \mathrm{MS}: \mathrm{m} / \mathrm{z}=630\left[\mathrm{M}^{+}\right]$; $\mathrm{CHN}$ calcd; $\mathrm{C}_{27} \mathrm{H}_{24} \mathrm{~F}_{2} \mathrm{~N}_{6} \mathrm{O}_{6} \mathrm{~S}_{2} ; \mathrm{C}, 51.42 ; \mathrm{H}, 3.84 ; \mathrm{N}, 13.33$; Found C, 51.40; H, 3.85; N, 13.30 .

1-ethyl-6,8-difluoro-7-(3-methyl-4-(5-((2-oxo-2-(ptolyl)ethyl)thio)-1,3,4-thiadiazol-2-yl)piperazin1-yl)-4-oxo-1,4-dihydroquinoline-3-carboxylic acid (5f)

IR (KBr) cm ${ }^{-1}: 3427$ (s, carboxylic, O-H str.), 2934(Ar. C-H str.), 2854(Ali. $\mathrm{CH}_{2}$ C-H str.), 1710(carboxylic, $\mathrm{C}=\mathrm{O}$ str.), 1623(ketonic, $\mathrm{C}=\mathrm{O}$ str.), 1576(Imine, $\mathrm{C}=\mathrm{N}$ str.), 1298(ethylic, C-H str.); ${ }^{1} \mathrm{H}-\mathrm{NMR}$ (DMSO-d6) $\delta p p m: 12.06(\mathrm{~s}, 1 \mathrm{H}$, carboxylic, $\mathrm{OH}), 8.84(\mathrm{~s}, 1 \mathrm{H}, \mathrm{H} 2$-quinoline), 7.54-7.68(m, $4 \mathrm{H}$, Ar.), 7.38(s, 1H, H5-quinoline), 4.64(s, 2H, $\left.\mathrm{CH}_{2}\right), 4.32$ (q, $\left.2 \mathrm{H}, \mathrm{NCH}_{2} \mathrm{CH}_{3}\right), 2.83-3.27(\mathrm{~m}, 7 \mathrm{H}$, piperazinyl), $2.37(\mathrm{~s}, 3 \mathrm{H}$, tolyl), $1.32\left(\mathrm{t}, 3 \mathrm{H}, \mathrm{NCH}_{2} \mathrm{CH}_{3}\right), 1.19\left(\mathrm{~s}, 3 \mathrm{H}\right.$, piperazinyl $\left.\mathrm{CH}_{3}\right)$; ${ }^{13} \mathrm{C}$ - NMR (DMSO-d6) Sppm: 188, 179, 158, 147, 135, 128, $116,108,68,35,16,7$.

\section{7-(4-(5-((2-(4-methoxyphenyl)-2-oxoethyl)thio)-} 1,3,4-thiadiazol-2-yl)-3-methylpiperazin-1-yl)1-ethyl-6,8-difluoro-4-oxo-1,4-dihydroquinoline3-carboxylic acid (5g)

IR (KBr) cm ${ }^{-1}$ : 3445(carboxylic, O-H str.), 3011(Ar. C-H str.), 2851(Ali. $\mathrm{CH}_{2}, \mathrm{C}-\mathrm{H}$ str.), 1728(carboxylic, $\mathrm{C}=\mathrm{O}$ str.), 1634(ketonic, $\mathrm{C}=\mathrm{O}$ str.), 1581(Imine, $\mathrm{C}=\mathrm{N}$ str.), 1310 (ethylic, C-H str.); ${ }^{1} \mathrm{H}-\mathrm{NMR}$ (DMSO-d6) Sppm: 12.83(s, carboxylic, $1 \mathrm{H}, \mathrm{OH}), 9.04(\mathrm{~s}, 1 \mathrm{H}, \mathrm{H} 2$-quinoline), 7.14-7.98(m, 4H, Ar.), 7.84(s, 1H, H5-quinoline), 4.71(s, 2H, $\left.\mathrm{CH}_{2}\right), 4.37(\mathrm{q}$, $\left.2 \mathrm{H}, \mathrm{NCH}_{2} \mathrm{CH}_{3}\right), 3.83(\mathrm{~s}, 3 \mathrm{H}$, methoxyl), 2.92-3.67(m, $7 \mathrm{H}$, piperazinyl), $1.31\left(\mathrm{t}, 3 \mathrm{H}, \mathrm{NCH}_{2} \mathrm{CH}_{3}\right), 1.11(\mathrm{~s}, 3 \mathrm{H}$, piperazinyl $\mathrm{CH}_{3}$ ); ${ }^{13} \mathrm{C}$ - NMR (DMSO-d6) Sppm: 191, 184, 168, 156, 138, 129, 112, 108, 60, 38, 18, 11; MS: m/z = $617[\mathrm{M}+1]$; $\mathrm{CHN}$ calcd; $\mathrm{C}_{28} \mathrm{H}_{27} \mathrm{~F}_{2} \mathrm{~N}_{5} \mathrm{O}_{5} \mathrm{~S} ; \mathrm{C}, 54.62 ; \mathrm{H}, 4.42 ; \mathrm{N}, 11.38$; Found $\mathrm{C}$, 54.64; H, 4.40; N, 11.37 . 7-(4-(5-((2-(4-aminophenyl)-2-oxoethyl)thio)1,3,4-thiadiazol-2-yl)-3-methylpiperazin-1-yl)1-ethyl-6,8-difluoro-4-oxo-1,4-dihydroquinoline3-carboxylic acid (5h)

IR (KBr) $\mathrm{cm}^{-1}$ : 3452(carboxylic, O-H str.), 3367(Ar. $\mathrm{NH}_{2}$, N-H str.), 3027(Ar. C-H str.), 2851(Ali. $\mathrm{CH}_{2}, \mathrm{C}-\mathrm{H}$ str.), 1736(carboxylic, $\mathrm{C}=\mathrm{O}$ str.), 1628 (ketonic, $\mathrm{C}=\mathrm{O}$ str.), 1580(Imine, $\mathrm{C}=\mathrm{N}$ str.), 1327 (ethylic, $\mathrm{C}-\mathrm{H}$ str.); ${ }^{1} \mathrm{H}-\mathrm{NMR}$ (DMSO-d6) Sppm: 12.88(s, carboxylic, 1H, OH), 9.12(s, 1H, H2-quinoline), 7.86(s, 1H, H5-quinoline), 6.83-7.72(m, $4 \mathrm{H}, \mathrm{Ar}$ ), 6.31(s, 2H, Ar. $\left.\mathrm{NH}_{2}\right), 4.72\left(\mathrm{~s}, 2 \mathrm{H}, \mathrm{CH}_{2}\right), 4.32(\mathrm{q}$, $\left.2 \mathrm{H}, \mathrm{NCH}_{2} \mathrm{CH}_{3}\right), 3.02-3.62(\mathrm{~m}, 7 \mathrm{H}$, piperazinyl), $1.29(\mathrm{t}, 3 \mathrm{H}$, $\left.\mathrm{NCH}_{2} \mathrm{CH}_{3}\right), 1.14\left(\mathrm{~s}, 3 \mathrm{H}\right.$, piperazinyl $\left.\mathrm{CH}_{3}\right) ;{ }^{13} \mathrm{C}-\mathrm{NMR}$ (DMSO-d6) dppm: 197, 187, 172, 151, 133, 123, 117, 107, 67, 35,$16 ; \mathrm{MS}: \mathrm{m} / \mathrm{z}=601[\mathrm{M}+1] ; \mathrm{CHN}$ calcd; $\mathrm{C}_{27} \mathrm{H}_{26} \mathrm{~F}_{2} \mathrm{~N}_{6} \mathrm{O}_{4} \mathrm{~S}_{2}$; C, 53.99; H, 4.36; N, 13.99; Found C, 54.02; H, 4.37; N, 13.98 .

\section{7-(4-(5-((2-(4-hydroxyphenyl)-2-oxoethyl)thio)-} 1,3,4-thiadiazol-2-yl)-3-methylpiperazin-1-yl)1-ethyl-6,8-difluoro-4-oxo-1,4-dihydroquinoline3-carboxylic acid (5i)

IR (KBr) cm cm $^{-1}$ 3544(phenolic, O-H str.), 3410(carboxylic, O-H str.),2973(Ar. C-H str.), 2850(Ali. $\mathrm{CH}_{2}, \mathrm{C}-\mathrm{H}$ str.), 1723(carboxylic, $\mathrm{C}=\mathrm{O}$ str.), 1662(ketonic, $\mathrm{C}=\mathrm{O}$ str.), 1575(Imine, $\mathrm{C}=\mathrm{N}$ str.), 1322 (ethylic, C-H str.); ${ }^{1} \mathrm{H}-\mathrm{NMR}$ (DMSO-d6) Sppm: 12.62(s, carboxylic, 1H, OH), 8.99(s, 1H, H2-quinoline), 7.79(s, 1H, H5-quinoline), 6.81-7.77(m, $4 \mathrm{H}, \mathrm{Ar}$ ), 5.35(s, $1 \mathrm{H}$, phenolic ), 4.73(s, $\left.2 \mathrm{H}, \mathrm{CH}_{2}\right), 4.37(\mathrm{q}$, $\left.2 \mathrm{H}, \mathrm{NCH}_{2} \mathrm{CH}_{3}\right), 2.94-3.37(\mathrm{~m}, 7 \mathrm{H}$, piperazinyl), $1.23(\mathrm{t}, 3 \mathrm{H}$, $\left.\mathrm{NCH}_{2} \mathrm{CH}_{3}\right), 1.08\left(\mathrm{~s}, 3 \mathrm{H}\right.$, piperazinyl $\left.\mathrm{CH}_{3}\right) ;{ }^{13} \mathrm{C}-\mathrm{NMR}(\mathrm{DM}-$ SO-d6) dppm: 193, 182, 175, 154, 130, 121, 115, 110, 71, $37,18$.

\section{7-(4-(5-((2-([1,1'-biphenyl]-4-yl)-2-oxoethyl)thio)-}

1,3,4-thiadiazol-2-yl)-3-methylpiperazin-1-yl)-

1-ethyl-6,8-difluoro-4-oxo-1,4-dihydroquinoline3-carboxylic acid (5j)

IR (KBr) $\mathrm{cm}^{-1}$ : 3462(carboxylic, O-H str.),3084(Ar. C-H str.), 2852(Ali. $\mathrm{CH}_{2}, \mathrm{C}-\mathrm{H}$ str.), 1733(carboxylic, $\mathrm{C}=\mathrm{O}$ str.), 1656(ketonic, $\mathrm{C}=\mathrm{O}$ str.), 1566(Imine, $\mathrm{C}=\mathrm{N}$ str.), 1337 (ethylic, C-H str.); ${ }^{1} \mathrm{H}-\mathrm{NMR}$ (DMSO-d6) $\delta p p m$ : 13.04(s, carboxylic, $1 \mathrm{H}, \mathrm{OH}), 9.10(\mathrm{~s}, 1 \mathrm{H}, \mathrm{H} 2$-quinoline $), 7.88(\mathrm{~s}, 1 \mathrm{H}$, H5-quinoline), 7.75-8.00(m, 4H, Ar.), 7.41-7.52(m, 5H, Ar.), 4.74(s, $\left.2 \mathrm{H}, \mathrm{CH}_{2}\right), 4.53\left(\mathrm{q}, 2 \mathrm{H}, \mathrm{NCH}_{2} \mathrm{CH}_{3}\right), 2.92-3.46(\mathrm{~m}, 7 \mathrm{H}$, piperazinyl), $1.27\left(\mathrm{t}, 3 \mathrm{H}, \mathrm{NCH}_{2} \mathrm{CH}_{3}\right), 1.11(\mathrm{~s}, 3 \mathrm{H}$, piperazinyl $\mathrm{CH}_{3}$ ); ${ }^{13} \mathrm{C}$ - NMR (DMSO-d6) Sppm: 197, 184, 179, 158, 133, 127, 121, 106, 65, 39, 21, 9; MS: m/z = $661\left[\mathrm{M}^{+}\right]$;CHN calcd; $\mathrm{C}_{33} \mathrm{H}_{29} \mathrm{~F}_{2} \mathrm{~N}_{5} \mathrm{O}_{4} \mathrm{~S}_{2} ; \mathrm{C}, 59.90 ; \mathrm{H}, 4.42 ; \mathrm{N}, 10.58$; Found $\mathrm{C}$, $59.92 ; \mathrm{H}, 4.41 ; \mathrm{N}, 10.60$. 


\section{DISCUSSION}

\section{Chemistry}

The synthesis of p-substituted phenacyl bromide $2(a-j)$ formation mechanism carried out by substitution acetophenone, acetic acid, and liquid bromine, reaction mechanism followed via acetic acid-mediated $\mathrm{H}^{+}$ions rather than lewis acid. Probably this reaction mechanism is followed by the bromination of the methyl group and it can be restricted to mono substitution, when the reaction is carried out in acidic media in presence of anhydrous $\mathrm{AlCl}_{3} \cdot{ }^{33}$ The synthesis of 2-amino-5-benzoylmethylenethio- 1,3,4-thiadiazole 3(a-j) was carried out by reacting $2(\mathrm{a}-\mathrm{j})$ and 2 -amino-5-mercapto1,3,4-thiadiazole via de-hydro-bromination mechanisms. In the reaction alkali hydroxyl ion abstract the mercapto proton, not the amino proton, because mercapto group having strong electron density centre on sulfur rather than amino nitrogen atom although nitrogen has strong electronegative element than sulfur electron density more on sulfur hence, the probability to abstract the proton of the mercapto group by hydroxyl ion is more where finding more electron density centre. Compounds $3(\mathrm{a}-\mathrm{j})$ were directly converted to 2-chloro-5-benzoylmethylenethio-1,3,4-thiadiazole $\quad 4(\mathrm{a}-\mathrm{i})$ by diazotization of amines followed by chlorination, which obeyed Sandmeyer reaction. This reaction is considered to be a more convenient method for introducing a halogen substituent at the desired position of an aromatic ring. The synthesis of [(7-(4--(5-sustituted-benzoylthio)-1,3,4-thiadiazol-2-yl)-3-methyl piperazine-1-yl)-1-ethyl-6,8-difluoro4-oxo-1,4-dihydroquinoline-3-carboxylic acid] 5(a-j) was based on aromatic nucleophilic substitution reaction mechanism involving substrate as 2-chloro-5-benzoylmethylenethio-1,3,4-thiadiazole 4(a-j) and fluoroquinolones with sodium bicarbonate in presence of $\mathrm{N}, \mathrm{N}$-dimethylformamide, (DMF) which seemed to be a convenient route to execute the synthesis of title compounds. ${ }^{34}$

\section{Microbiological assay $\mathbf{5 ( a - j )}$}

\section{Antibacterial activity}

The synthesized compounds 5 (a-j) explored In-vitro antibacterial activity against two Gram-positive and two Gramnegative strains i.e. Staphylococcus aureus, Bacillus subtilis and Escherichia coli, Pseudomonas aeruginosa respectively to determine the MICs, which gave profound conclusion about the most active analogues. As per results depicted in compounds, classified the active and non-active analogues based on screened standard drug's MICs. According to that, 1.33 to 2.50 categorized highly active; $2.50-10.0$ considered to be moderately active and $>10.0$ deemed to be least active when compared with that of standards gatifloxacin.

Largely, most of the synthesized derivatives were found progressive to moderate activity against the Gram-positive bacterial strains of the lomefloxacin series. However, methyl and methoxy substituted (9f \& 9g) of lomefloxacin derivatives were found to be the least active ( 15.33 to $17.00 \mu \mathrm{g} /$ $\mathrm{ml}$ ) against gram-positive bacterial strains of Staphylococcus aureus and Bacillus subtilis. Apart from that, none of the derivatives was found highly active against Escherichia coli and Pseudomonas aeruginosa gram-negative strains.

\section{Antitubercular activity}

The derivatives were screened for In-vitro antitubercular against $H_{37} R v$ strain, using micro Alamar blue reagent, in this assay, blue colour converted into red colour indicates the test compound would be non-active up to that particular concentration. This assay has conducted based on BACTEC radiometric method. MicroplateAlamar blue assay (MABA) has certain advantages over the conventional disc diffusion method $^{35}$. In this assay, eight double-fold serially diluted concentrations were taken between 0.8 to $100 \mu \mathrm{g} / \mathrm{ml}$. In which $(-\mathrm{Cl})$ substituted of lomefloxacin9b derivatives were found to be an excellent activity $(0.8 \mu \mathrm{g} / \mathrm{ml})$ which is comparable to that of standards $(0.8 \mu \mathrm{g} / \mathrm{ml})$.

\section{In-vitro toxicity study}

The potent antitubercular agents were subjected to toxicity study with the help of MTT assay on Hep-G2 normal liver cell line. The value of PI is unitless and a higher value is considered to be the most efficient agent. The compound $5 \mathrm{~b}$ (-Cl substituted derivative) shown PI value 68.75 , indicated more toxic as compared to reference rifampicin as its PI value was 81.25 . Hence these derivatives have not secured the safe candidate, as the PI value is very low as compared to reference Rifampicin. The present nucleus permits various modifications at the site of the desire position to obtain more derivatives with improve pharmacokinetics and pharmacodynamics profiles.

\section{CONCLUSIONS}

C-7 position of fluoroquinolone nucleus permits the bulkier substitutions as Benzoylmethylenethio-1, 3, 4-thiadiazole (5 a-j) provide satisfactory yields by aromatic nucleophilic substitution reaction $\left(\mathrm{SN}^{2}\right)$. The substituted derivatives of fluoroquinolone were moderate to least active against Grampositive bacteria and M. tuberculosis pathogen. However, all the synthesized derivatives did not respond to the gram-negative strains. So that, these practical results offering numerous modifications in the same scaffold, to develop the new chemical entity.

\section{ACKNOWLEDGEMENTS}

The authors acknowledge the immense help received from the scholars whose articles are cited and included in the ref- 
erences of this manuscript. The authors are also grateful to the authors, editors, and publishers of all the articles, journals, and books that have been reviewed and discussed in this article.

\section{Source of Funding: Nil}

\section{Conflict of Interest: Nil}

Authors' Contribution: Agrawal Kapil, Sheelpriyawalde and Ittadwar Abhay has done the design of the work, Data collection, Data analysis and interpretation.

\section{REFERENCES}

1. Wolfson JS, Hooper DC. The Fluoroquinolones: Structures, Mechanisms of Action and Resistance, and Spectra of Activity In-Vitro. AntimicrobagentsandChemother., 1985 Oct 28(4); 581-6.

2. Ball P. Bacterial resistance to fluoroquinolones: Lessons to be learned', Infection, 1994; 22(2 Supplement).

3. Domagala JM.1-Substituted 7-[3-[(Ethylamino)methyl]1-pyrrolidinyl]-6,8-difluoro-1,4-dihydro-4-oxo-3-quinolinecarboxylic Acids. New Quantitative Structure-Activity Relationships at N1 for the Quinolone Antibacterials', J. Med Chem., 1988; 31(5):991-1001.

4. Chu DT, Fernandes PB, Claiborne AK, Pernet AG. Synthesis and structure-activity relationships of novel arylfluoroquinolone antibacterial agents. J. Med Chem.,1985; 28(11):1558-64

5. Rosen T. Mechanism of inhibition of DNA gyrase by quinolone antibacterials: A cooperative drug-DNA binding model. Biochem. 1989; 28(9):3886-94.

6. Sarro A, Sarro G. Adverse Reactions to Fluoroquinolones. An Overview on Mechanistic Aspects. Curr Med Chem. 2001; 8(4):371-84.

7. Anderson V, Osheroff N. Type II Topoisomerases as Targets for Quinolone Antibacterials Turning Dr. Jekyll into Mr. Hyde. Curr Pharm Design. 2001;7(5):337-53..

8. Koga H, Itoh A, Murayama S, Suzue S, IrikuraT. StructureActivity Relationships Of Antibacterial 6, 7-and 7, 8-Disubstituted 1-Alkyl-1, 4-Dihydro-4-Oxoquinoline-3-Carboxylic Acids. J. Med. Chem, 1980; 23(12):1358-63.

9. Hagihara M, Kashiwase H, Katsube T, Kimura T, Komai T, Momota K, Shimada K. Synthesis and anti-HIV activity of arylpiperazinyl fluoroquinolones: A new class of anti-HIV agents. Bioorg \& Med. Chem. Letters, 1999;9(21):3063-68.

10. Domagala JM, Hanna LD, Heifetz CL, Hutt MP, Mich TF, Sanchez J. New structure-activity relationships of the quinolone antibacterials using the target enzyme. The development and application of a DNA gyrase assay. J. Med. Chem., 1986;29(3):394-404.

11. Kondo H, Sakamoto F, Kodera Y, Tsukamoto G. Studies on prodrugs. 5. Synthesis and antimicrobial activity of $\mathrm{N}$-(oxoalkyl) norfloxacin derivatives. J Med. Chem. 1986;29(10):2020-24.

12. Fang KC, Chen YL, Sheu JY, Wang TC, Tzeng CC. Synthesis, Antibacterial, and Cytotoxic Evaluation of Certain 7-Substituted Norfloxacin Derivatives. J. Med. Chem., 2000;43(20):3809-12.

13. Gootz TD, McGuirk PR, Moynihan MS, Haskell SL, Placement of alkyl substituents on the C-7 piperazine ring of fluoroquinolones: dramatic differential effects on mammalian topoisomerase II and DNA gyrase. Antimicrob Age Chemother., 1994;38(1):130-33.
14. Pandeya, S. N., Sriram, D., Nath, G., \& De Clercq, E., Synthesis, antibacterial, antifungal and anti-HIV activities of norfloxacin Mannich bases. Euro J. Med. Chem, 2000;35(2):249-55

15. Chu, D. T. W., Fernandes, P. B., Maleczka, R. E., Nordeen, C. W., \& Pernet, A. G., Synthesis and structure-activity relationship of 1-aryl-6,8-difluoroquinolone antibacterial agents. J. Med. Chem., 1987;30(3):504-09.

16. Shen, L. L., \& Pernet, A. G., Mechanism of inhibition of DNA gyrase by analogues of nalidixic acid: the target of the drugs is DNA. Proc Natl Acad Sci U S A, 1985; 82(2):307-11.

17. L. L. Shen, W E. Kohlbrenner,D. W. J. Baranowski; Mechanism of quinolone inhibition of DNA gyrase: Appearance of unique norfloxacin binding sites in enzyme-DNA complexes; J Biol Chem., 1989 Feb; 264(5):2973-78.

18. Shen, L. L., Baranowski, J., \& Pernet, A. G., Mechanism of inhibition of DNA gyrase by quinolone antibacterials: specificity and cooperativity of drug binding to DNA. Biochemistry, 1989; 28(9):3879-85.

19. Klopman, G., Macina, O. T., Levinson, M. E., \& Rosenkranz, H. S., Computer automated structure evaluation of quinolone antibacterial agents. Antimicrob agents and Chemother., 1987;31(11):1831-40.

20. Russo, F.; Santagati, M, Synthesis and evaluation of the antibacterial activity of benzothiazole derivatives of 1,3,4- thiadiazole and imidazo/2,1-b,1,3,4-thiadiazole Il Farmaco Sci., 1976; 31:41-48.

21. Tsotinis A, Varvaresou A, Calogeropoulou T, Siatra-Papastaikoudi T, Tiligada A; Synthesis and antimicrobial evaluation of indole containing derivatives of 1,3,4-thiadiazole, 1,2,4-triazole and their open-chain counterparts, Arzneimittelforschung, 01 Mar 1997; 47(3):307-10.

22. Organic Syntheses, Coll, R. M. Cowper, L. H. Davidson, and A. H. Blatt, Eds., vol. 2, pp. 480-481, John Wiley \& Sons, NewYork, NY, USA, 1943.

23. Organic Syntheses. Coll, G. H. Coleman, G. E. Honeywell, and A. H. Blatt, Eds., vol. 2, pp. 443-445, John Wiley \& Sons, New York, NY, USA, 1943.

24. Dogan H.N, Duran A, Rollas S, Sener G, Uysal MK, Gülen D. Synthesis of new 2,5-Disubstituted-1,3,4-thiadiazoles and preliminary evaluation of anticonvulsant and antimicrobial activities. Bioorg Med Chem., 2002; 10(9):2893-98.

25. Varvaresou A, Sinatra-Papastaikoudi T, Tsotinis A, Tsantili-Kakoulidou A, Vamvakides, A. Synthesis, lipophilicity and biological evaluation of indole-containing derivatives of 1,3,4-thiadiazole and 1,2,4-triazole. Il Farmaco, 1998;53(5):320-26.

26. Foroumadi A, Rineh A, Emami S, Siavoshi F, Massarra S, Safari F.et al. Synthesis and anti-Helicobacter pylori activity of 5-(nitroaryl)-1,3,4-thiadiazoles with certain sulfur-containing alkyl side chain.Bioorg. Med Chem Lett. 2008; 18(11):3315-20.

27. Foroumadi A. Emami S, Pournourmohammadi S, Kharazmi A, Shafiee A. Synthesis and in vitro leishmanicidal activity of 2-(1-methyl-5-nitro-1H-imidazol-2-yl)-5-substituted-1, 3, 4-thiadiazole derivatives. Eur J Med Chem. 2005; 40(12):1346-50.

28. Foroumadi A, Mansouri S, Kiani Z, Rahmani A. Synthesis and in vitro antibacterial evaluation of N-[5-(5-nitro-2-thienyl)-1, 3, 4-thiadiazole-2-yl] piperazinyl quinolones. Eur. J Med Chem. 2003; 38(9):851-54.

29. GotoS, Sakamoto H, Ogawa M. Bactericidal activity of cefazolin, cefoxitin, and cefmetazole against Escherichia coli and Klebsiellapneumoniae. Chemother. 1982; 28(1):18-25.

30. SulingWJ, SeitzLE\& Pathak V. Antimycobacterial activities of 2,4-diamino-5-deazapteridine derivatives and effects on mycobacterial dihydrofolatereductase, Antimicrob agent Chemother. 2000;44(10):2784-2793. 
31. Yajko DM, MadejJJ\& Lancaster MV. Colourimetric method for determining MICs of antimicrobial agents for Mycobacterium tuberculosis. J Clin Micro. 1995; 33(9):2324-27.

32. Sriram D, Yogeeswari P, ReddySP. Synthesis of pyrazinamide Mannich bases and its antitubercular properties. Bioorg Med Chem Letters, 2006;16(8):2113-16.
33. Morrison RT, Boyd RN. Organic Chemistry, Pearson Education, New Delhi, India:2004.

34. Agrawal KM, Talele GS. Synthesis and antibacterial, antimycobacterial and docking studies of novel N-piperazinyl fluoroquinolones. Med.Chem Res. 2013; 22(2):818-31.

Table 1: Physicochemical data of synthesized compound (5a-5j)

\begin{tabular}{lllllcc} 
Compound No & $\mathrm{R}$ & Nature & Molecular formula & Molecular mass & $\begin{array}{c}\text { Melting } \\
\text { point }\left({ }^{\circ} \mathbf{C}\right)\end{array}$ & $\begin{array}{c}\text { Yield } \\
(\%)\end{array}$ \\
\hline $5 \mathrm{a}$ & $-\mathrm{H}$ & Amorphous & $\mathrm{C}_{27} \mathrm{H}_{25} \mathrm{~F}_{2} \mathrm{~N}_{5} \mathrm{O}_{4} \mathrm{~S}_{2}$ & 585 & 215 & 54 \\
$5 \mathrm{~b}$ & $-\mathrm{Cl}$ & Amorphous & $\mathrm{C}_{27} \mathrm{H}_{24} \mathrm{~F}_{2} \mathrm{ClN}_{5} \mathrm{O}_{4} \mathrm{~S}_{2}$ & 619 & 245 & 50 \\
$5 \mathrm{C}$ & $-\mathrm{Br}$ & Amorphous & $\mathrm{C}_{27} \mathrm{H}_{24} \mathrm{~F}_{2} \mathrm{BrN}_{5} \mathrm{O}_{4} \mathrm{~S}_{2}$ & 664 & 250 & 52 \\
$5 \mathrm{~d}$ & $-\mathrm{F}$ & Amorphous & $\mathrm{C}_{27} \mathrm{H}_{24} \mathrm{~F}_{3} \mathrm{~N}_{5} \mathrm{O}_{4} \mathrm{~S}_{2}$ & 603 & 185 & 55 \\
$5 \mathrm{e}$ & $-\mathrm{NO}_{2}$ & Amorphous & $\mathrm{C}_{27} \mathrm{H}_{24} \mathrm{~F}_{2} \mathrm{~N}_{6} \mathrm{O}_{6} \mathrm{~S}_{2}$ & 630 & 240 & 48 \\
$5 \mathrm{f}$ & $-\mathrm{CH}_{3}$ & Amorphous & $\mathrm{C}_{28} \mathrm{H}_{27} \mathrm{~F}_{2} \mathrm{~N}_{5} \mathrm{O}_{4}$ & 599 & 228 & 46 \\
$5 \mathrm{~S}$ & $-\mathrm{OCH}_{3}$ & Amorphous & $\mathrm{C}_{28} \mathrm{H}_{27} \mathrm{~F}_{2} \mathrm{~N}_{5} \mathrm{O}_{5}$ & 615 & 230 & 52 \\
$5 \mathrm{~S}$ & $-\mathrm{NH}_{2}$ & Amorphous & $\mathrm{C}_{27} \mathrm{H}_{26} \mathrm{~F}_{2} \mathrm{~N}_{6} \mathrm{O}_{4} \mathrm{~S}_{2}$ & 600 & 220 & 58 \\
$5 \mathrm{i}$ & $-\mathrm{OH}$ & Amorphous & $\mathrm{C}_{27} \mathrm{H}_{25} \mathrm{~F}_{2} \mathrm{~N}_{5} \mathrm{O}_{5} \mathrm{~S}_{2}$ & 601 & 240 & 52 \\
$5 \mathrm{j}$ & $-\mathrm{C}_{6} \mathrm{H}_{5}$ & Amorphous & $\mathrm{C}_{33} \mathrm{H}_{29} \mathrm{~F}_{2} \mathrm{~N}_{5} \mathrm{O}_{4}$ & 661 & 248 & 54 \\
\hline
\end{tabular}

Table 2: Antibacterial and antimycobacterial activity against the screened compounds along with In-vitro toxicity studies $\mathbf{5}(\mathbf{a}-\mathbf{j})$.

\begin{tabular}{|c|c|c|c|c|c|c|c|}
\hline $\begin{array}{l}\text { Compound } \\
\text { Number }\end{array}$ & $\begin{array}{l}\text { bS. A. G(+) } \\
{ }^{a} \mathbf{M I C}\end{array}$ & $\begin{array}{c}\text { bB. S. G(+) } \\
{ }^{\text {a MIC }}\end{array}$ & 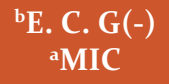 & $\begin{array}{c}\text { bP. A. G(-) } \\
{ }^{a} \text { MIC }\end{array}$ & $\begin{array}{l}{ }^{\mathrm{c}} \mathrm{M} . \mathrm{TB} \\
{ }^{\mathrm{a}} \mathrm{MIC}\end{array}$ & ${ }^{\mathrm{d}} \mathrm{I} \mathrm{C}_{50}$ & eSI \\
\hline $5 \mathrm{a}$ & 15.67 & 17.00 & 31.33 & 65.33 & Resist & -- & -- \\
\hline $5 b$ & 4.33 & 4.33 & 15.33 & 31.67 & 0.8 & 55 & 68.75 \\
\hline $5 \mathrm{C}$ & 8.33 & 7.67 & 16.33 & 17.00 & 3.12 & -- & -- \\
\hline $5 \mathrm{~d}$ & 8.32 & 5.94 & 5.33 & 4.45 & 1.60 & -- & -- \\
\hline 5e & 4.00 & 7.00 & 15.67 & 16.00 & 3.12 & -- & -- \\
\hline $5^{f}$ & 15.67 & 17.00 & 31.00 & 64.33 & Resist & -- & -- \\
\hline $5 \mathrm{~g}$ & 15.67 & 15.33 & 66.00 & 64.67 & 12.5 & -- & -- \\
\hline $5 \mathrm{~h}$ & 4.67 & 4.67 & 16.33 & 33.00 & 12.5 & -- & -- \\
\hline $5 \mathrm{i}$ & 4.67 & 6.67 & 31.67 & 32.67 & Resist & -- & -- \\
\hline $5 j$ & 3.00 & 2.33 & 8.67 & 8.67 & 3.12 & -- & -- \\
\hline GATI* & 1.33 & 2.00 & 7.67 & 3.67 & -- & -- & -- \\
\hline RIP* & -- & -- & -- & -- & 0.8 & 65 & 81.25 \\
\hline
\end{tabular}

a :-MIC: Minimum inhibitory concentration (in $\mu \mathrm{g} / \mathrm{ml}$ ) required to inhibit $90 \%$ inhibition against M. TB and antibacterial.

b :-As a bacteria;SA:- S. aureus(Gram positive), BS:- B. subtilis(Gram positive);

EC:- E. Coli (Gram negative), PA:-P. aeruginosa(Gram negative)

${ }^{\mathrm{C}} \mathrm{M}$. TB:- Mycobacterium Tuberculosis

d:- IC5o inhibition concentration (inhibited $50 \%$ of total cells in $1 \mathrm{M}$ and converted to $\mu \mathrm{g} / \mathrm{mL}$ for SI calculation)

*:- As reference pure drug :- GATI: Gatifloxacin; RIP: rifampicin.

e:- SI Index selectivity index (ratio between $\mathrm{IC}_{50}$ and M. tuberculosis MIC value) 
<smiles>CCn1cc(C(=O)O)c(=O)c2cc(F)c(N3CCNCC3)cc21</smiles>

Norfloxacin<smiles>COc1c(N2CCNC(C)C2)c(F)cc2c(=O)c(C(=O)O)cn(C3CC3)c12</smiles>

Gatifloxacin<smiles>O=C(O)c1cn(C2CC2)c2cc(N3CCNCC3)c(F)cc2c1=O</smiles>

Ciprofloxacin<smiles>CC1CN(c2c(F)c(N)c3c(=O)c(C(=O)O)cn(C4CC4)c3c2F)CC(C)N1</smiles>

Figure 1<smiles>[R]c1ccc(C(=O)Sc2nnc(N3CCN(c4c(F)cc5c(=O)c(C(=O)O)cn(CC)c5c4F)CC3C)s2)cc1</smiles>

Figure 2: Targeted Derivative.
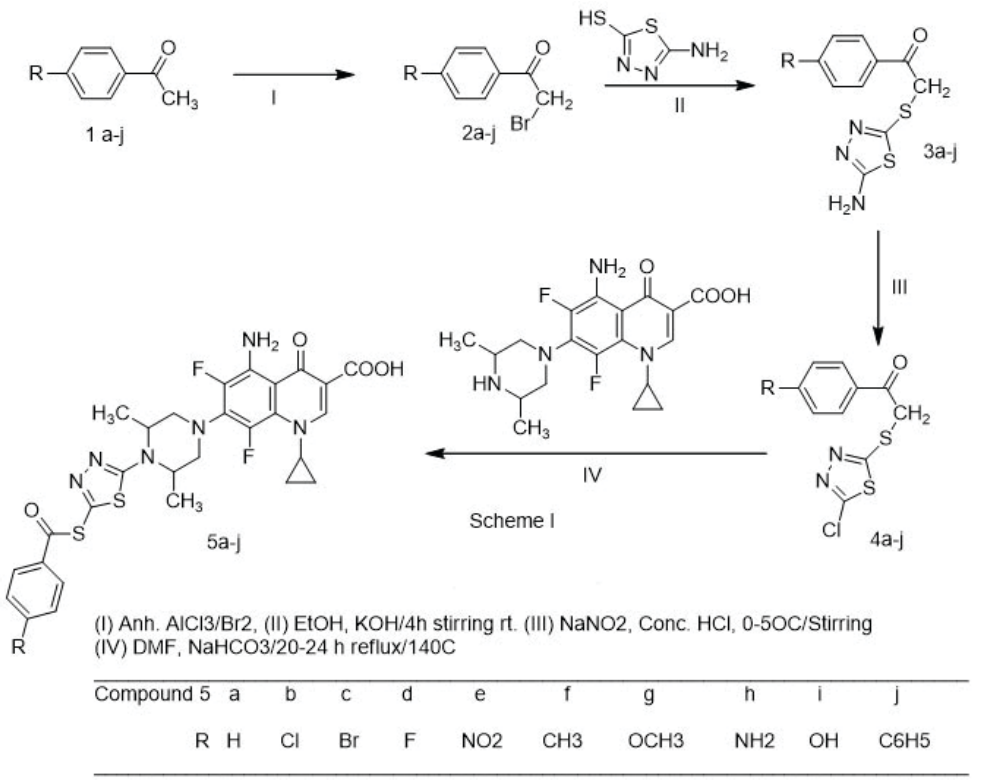

Figure 3: Synthetic Scheme. 